\title{
Feasibility of using apparent thermophysical properties to incorporate the effect of blood perfusion in estimation of temperature in tissues
}

\author{
Mohammed SHURRAB*, Haidong WANG*, Takanobu FUKUNAGA*, Kosaku KURATA* and \\ Hiroshi TAKAMATSU* \\ *Department of Mechanical Engineering, Kyushu University \\ E-mail: takamatsu@mech.kyushu-u.ac.jp
}

Received: 7 August 2017; Revised: 19 September 2017; Accepted: 10 October 2017

\begin{abstract}
Estimation of temperature distribution in tissues and organs is critically important for treatments such as hyperthermia, radiofrequency ablation and cryosurgery which expose malignant tissue to extreme temperatures that are different from the physiological temperature. Commonly, the bioheat equation, instead of heat conduction equation, is used for estimation to incorporate the effect of blood perfusion, because the heat transfer in tissues is significantly affected by blood perfusion in addition to thermophysical properties of tissues. Nevertheless, in many cases, the rate of blood perfusion is not available for human tissues and organs. This study therefore aims to examine if we can use the normal heat conduction equation with apparent thermophysical properties to take the effect of blood perfusion into account. Feasibility was checked by comparing the results obtained from the heat conduction equation and the bioheat equation. The result indicated that the simulation with the apparent thermal conductivity or specific heat capacity does not agree well with the temperature distribution inside a tissue with blood perfusion. However, the apparent thermal conductivity was useful to estimate the size of growing ice ball produced during cryosurgery.
\end{abstract}

Keywords : Apparent thermal conductivity, Apparent specific heat capacity, Blood perfusion, Bioheat equation, Cryosurgery

\section{Introduction}

Prediction of temperature distribution in tissues and organs is important for medical treatments such as hyperthermia (Oleson et al., 1989), radiofrequency ablation (Jain and Wolf, 2000), high-frequency focused ultrasound (Maass-Moreno et al., 1996) and cryosurgery (Shurrab et al., 2016), which induce a significant temperature change from the physiological state. However, precise data of thermal transport properties of human tissues are not available because of the lack of non-invasive technique for the measurement while the data measured in-vivo has been reported for animal tissues (Bowman, 1984; Newman and Lele, 1985). Furthermore, thermal transport in tissues is significantly affected by blood flow. Hence for the estimation of temperature inside tissues, intrinsic thermophysical properties and the rate of blood perfusion are needed.

The Pennes bioheat equation was the first that took into account the contribution of blood flow to the heat transport in tissues to reproduce the measured temperature profile in a human forearm (Pennes, 1948). The effect of blood flow in capillaries was incorporated into a heat source that includes the blood flow rate per unit volume of tissue as a parameter. The advantage of the Pennes model is that the perfusion term is simple and in proportion to the difference between the tissue temperature and the temperature of arterial blood that is assumed to be 
constant in the whole region (Diller et al., 1999; Valvano, 2006). However, the model has some controversial issues from physical points of view. The blood perfusion term is based on the heat balance within the whole system and thus is inconsistent with the conduction term that is derived from a local control volume. Furthermore, the model aimed at expressing the effect of a microvascular system which was however demonstrated afterwards to be thermally insignificant (Chato, 1980). These were the motivations of a number of subsequent studies that proposed different models for heat transport in living tissues. For example, Chen and Holmes divided the tissue into two parts, i.e. the part of thermally significant large blood vessels and the solid tissue part that includes the effect of isotropic small blood vessels (Chen and Holmes, 1980). Weinbaum and Jiji proposed a model that incorporates the effect of counter-flow heat transfer between parallel artery and vein based on the anatomical observation (Weinbaum et al., 1984; Jiji et al., 1984; Weinbaum and Jiji, 1985). The heat transport between a single vessel and surrounding tissue (Chato, 1980) or the heat transfer between parallel blood vessels (Lemons et al., 1987; Baish, 1990; Mooibroek and Lagendijk, 1991; Huang, 1996) were also examined.

Most of the models proposed after Pennes intended to incorporate the effect of thermally significant vessels that were neglected in the Pennes model. However, the models were much complicated than the original Pennes model and required much more information associated with the blood vessels and blood flow which is not available in many cases. In addition, the estimation from these models was not significantly different from the Pennes equation that was practically used for a rat liver (Valvano et al., 1984) and a pig kidney (Xu, 1991). Consequently, the Pennes bioheat equation has still been used for a number of applications (Zhao et al., 2005; Dehghan and Sabouri, 2012; Shahnazari et al., 2013) assuming constant blood perfusion (Li et al., 2002; Zhao et al., 2007) or temperature-dependent perfusion rate (Sanyal and Maji, 2001; Gang et al., 2007). However, in many cases for human tissues, even the blood perfusion rate is not available and is dependent on tissue types (Shitzer and Eberhart, 1985).

Considering the fact that both intrinsic thermophysical properties and the rate of blood perfusion are not available in many cases, we came up with a new practical idea of using the apparent thermal properties in the heat conduction equation instead of solving the bioheat equation. This study therefore aims to examine the feasibility of the idea by comparing the result of solving the bioheat equation and the normal heat conduction equation expressed with the apparent specific heat capacity or the apparent thermal conductivity. The case examined in this study was freezing of tissue around a cryoprobe during cryosurgery. The case with no phase change was also examined for the same model.
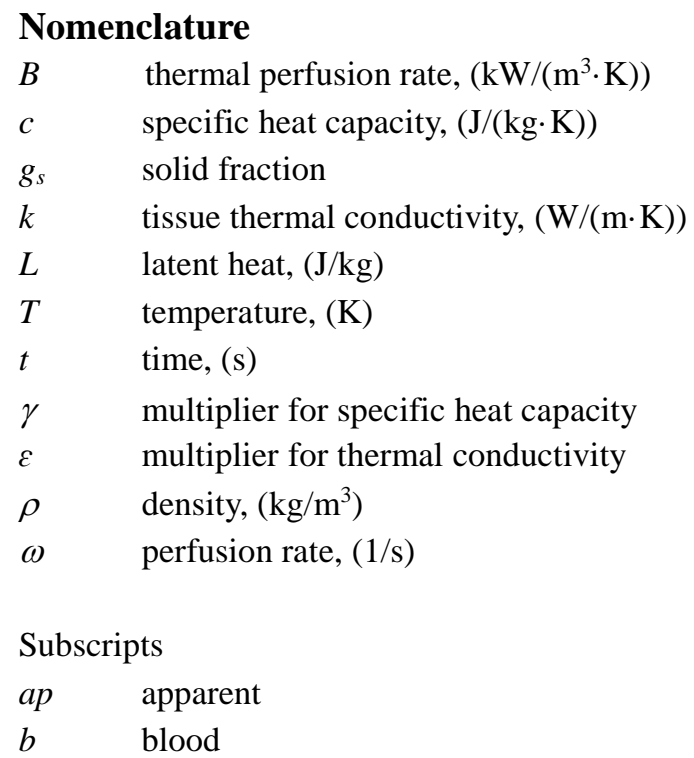


\section{Methods}

The effect of blood perfusion was mainly examined with a simulation of cryosurgery as a potential application, where a tissue is frozen with a 1.5-mm-dia. cryoprobe that is uniformly cooled at the surface $32 \mathrm{~mm}$ from the tip (Fig. 1). We solved the following Pennes bioheat equation taking freezing phenomena into account using an apparent heat capacity model (Shurrab et al., 2016):

$$
\rho\left(c-L \frac{\partial g_{s}}{\partial T}\right) \frac{\partial T}{\partial t}=\frac{1}{r} \frac{\partial}{\partial r}\left(k r \frac{\partial T}{\partial r}\right)+\frac{\partial}{\partial z}\left(k \frac{\partial T}{\partial z}\right)+\rho_{b} c_{b} \omega_{b}\left(T_{b}-T\right)
$$

where $T$ is the temperature $[\mathrm{K}], \rho$ is the density $\left[\mathrm{kg} / \mathrm{m}^{3}\right], c$ is the heat capacity $[\mathrm{J} /(\mathrm{kg} \cdot \mathrm{K})], k$ is the thermal conductivity $[\mathrm{W} /(\mathrm{m} \cdot \mathrm{K})], L$ is the latent heat $[\mathrm{J} / \mathrm{kg}], g_{s}$ is the solid fraction defined by the ratio between the mass of ice and the total mass, and $\omega$ is the rate of blood perfusion [1/s]. Subscript $b$ stands for the blood. The assumptions made were: (1) the effect of metabolic heat generation is negligible, (2) the tissue is considered as an isotonic sodium chloride aqueous solution, (3) freezing starts at $-0.5^{\circ} \mathrm{C}\left(g_{s}=0\right)$ and completes at $-21.1^{\circ} \mathrm{C}\left(g_{s}=1\right)$ following the phase diagram in the equilibrium state with no diffusion, and (4) blood perfusion does not exist ( $\omega_{b}$ $=0$ ) in the partially and completely frozen region. The thermophysical properties were estimated as a function of temperature taking into account the concentration of sodium chloride. The thermophysical properties of the partially frozen part $\left(-21.1^{\circ} \mathrm{C}<\mathrm{T}<-0.5^{\circ} \mathrm{C}\right)$ were treated as a mixture of pure ice and unfrozen liquid at the equilibrium mass fraction of sodium chloride (see (Shurrab et al., 2016) for the details).

Based on our experiment (Shurrab et al., 2016) using a Joule-Thomson cryoprobe, CryoHit ${ }^{\mathrm{TM}}$ Needle I (Gallil Medical), we assumed a cooling curve at the surface of cryoprobe as a boundary condition (Fig. 2). The solution was obtained for hypothesized thermal perfusion rate $B\left(=\rho_{b} c_{b} \omega_{b}\right)$ at three different boundary conditions, i.e. temperature change to $-70^{\circ} \mathrm{C},-90^{\circ} \mathrm{C}$ and $-100^{\circ} \mathrm{C}$, which was obtained by the experiment at supplied gas pressures of 22.4 MPa, 25.2 MPa, and 27.4 MPa, respectively.

To compare the temperature distribution with that simulated using Eq. (1), we solved the following normal heat conduction equation with the hypothesized apparent thermal conductivity $k_{a p}$ or the apparent specific heat capacity $c_{a p}$.

$$
\begin{aligned}
& \rho\left(c-L \frac{\partial g_{s}}{\partial T}\right) \frac{\partial T}{\partial t}=\frac{1}{r} \frac{\partial}{\partial r}\left(k_{a p} r \frac{\partial T}{\partial r}\right)+\frac{\partial}{\partial z}\left(k_{a p} \frac{\partial T}{\partial z}\right) \\
& \rho\left(c_{a p}-L \frac{\partial g_{s}}{\partial T}\right) \frac{\partial T}{\partial t}=\frac{1}{r} \frac{\partial}{\partial r}\left(k r \frac{\partial T}{\partial r}\right)+\frac{\partial}{\partial z}\left(k \frac{\partial T}{\partial z}\right)
\end{aligned}
$$

We did not examine the case of using both apparent thermal conductivity and apparent heat capacity. This is because there is no theoretical background for determining two parameters at the same time.

A commercial software (COMSOL Multiphysics) was used to obtain the numerical solution. Considering that a typical value of the blood perfusion rate $B$ is $17 \mathrm{~kW} /\left(\mathrm{m}^{3} \cdot \mathrm{K}\right)\left(\rho_{b}=1000 \mathrm{~kg} / \mathrm{m}^{3}, \omega=0.0051 / \mathrm{s}\right.$ and $c_{b}=3391$ $\mathrm{J} / \mathrm{kg} \cdot \mathrm{K}$ ) in soft tissues in previous studies (Zhao et al., 2007; Duck, 1990; Seyde et al., 1985; Koehler et al., 1985) we assumed $B$ as a parameter ranging from 0 to $50 \mathrm{~kW} /\left(\mathrm{m}^{3} \cdot \mathrm{K}\right)$. The given apparent thermal conductivity and heat capacity were expressed by the ratio to that of tissue by $\varepsilon\left(=k_{a p} / k\right)$ and $\gamma\left(=c_{a p} / c\right)$, respectively. Before simulation of freezing, two cases with no phase change were also examined to take an overview. One was the case where the probe temperature was suddenly decreased to $7{ }^{\circ} \mathrm{C}$ and the other was the case where the probe was uniformly 
heated at a constant heat flux.

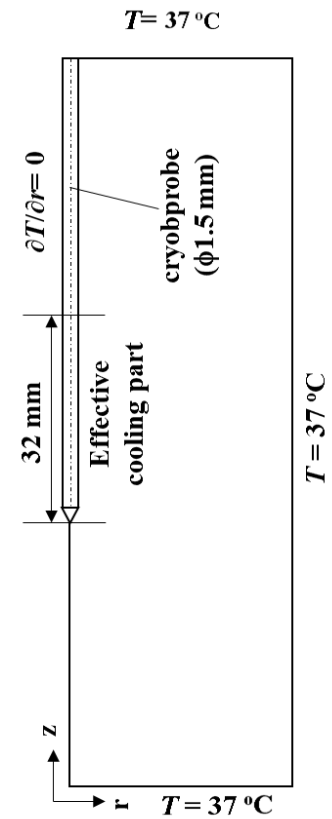

Fig. 1 Physical model.

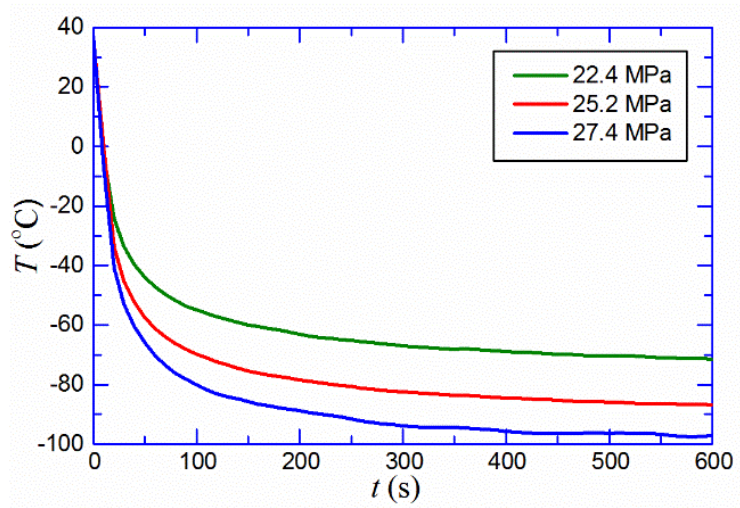

Fig. 2 Temperature change at the surface of cryoprobe given as the boundary condition.

\section{Results}

\subsection{Cooling or heating with no phase change}

Figure 3 shows the temperature distribution around the probe at $600 \mathrm{~s}$ after the probe surface was cooled to $7^{\circ} \mathrm{C}$, and compares the effect of blood perfusion with that of apparent thermal conductivity (Fig. 3(a)) and apparent heat capacity (Fig. 3(b)). At larger perfusion rate the temperature change becomes more significant near the probe. The increase of the heat capacity also narrows the thermally affected region, and the increase by a factor of four and nine has almost the same effect with the perfusion rate of 20 and $50 \mathrm{~kW} /\left(\mathrm{m}^{3} \cdot \mathrm{K}\right)$, respectively. In contrast, the increase of the thermal conductivity reduces the temperature gradient and extends the thermally affected region. Hence the enhancement of heat transport by blood perfusion is not equivalent to the increase of the apparent thermal conductivity. 

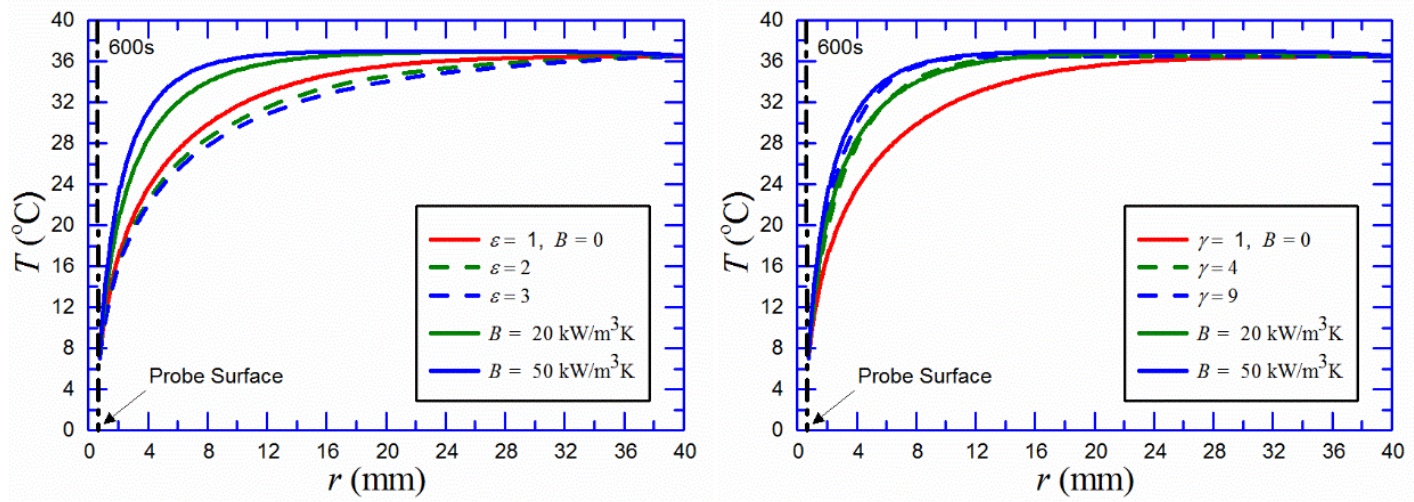

(a) Comparison with different apparent thermal conductivity (b) Comparison with different apparent heat capacity

Fig. 3 Radial temperature distribution at different rate of blood perfusion compared with the result of given apparent thermophysical properties in the case of cooling to $7^{\circ} \mathrm{C}$.

The effect of blood perfusion and the apparent heat capacity, which was similar in Fig. 3, was also examined for a given heat flux at the probe surface as the boundary condition. Figure 4 shows the temperature distribution after heating the probe at $10 \mathrm{~kW} / \mathrm{m}^{2}$ for a given value of blood perfusion and apparent heat capacity. The temperature at the surface increases due to heating at the constant heat flux. However, the blood perfusion suppresses the temperature increase and makes the temperature distribution approach a steady state earlier at higher perfusion rates. In contrast, without blood perfusion, the temperature keeps increasing and thus the heat penetrates deeper with time by heat conduction.

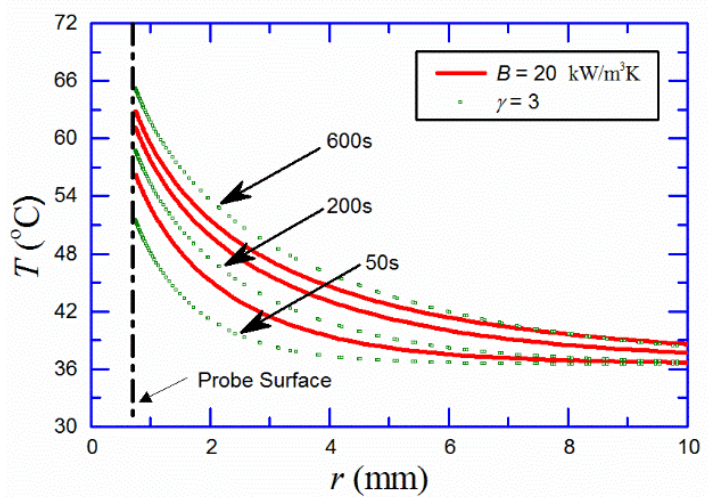

(a) $B=20 \mathrm{~kW} /\left(\mathrm{m}^{3} \cdot \mathrm{K}\right)$

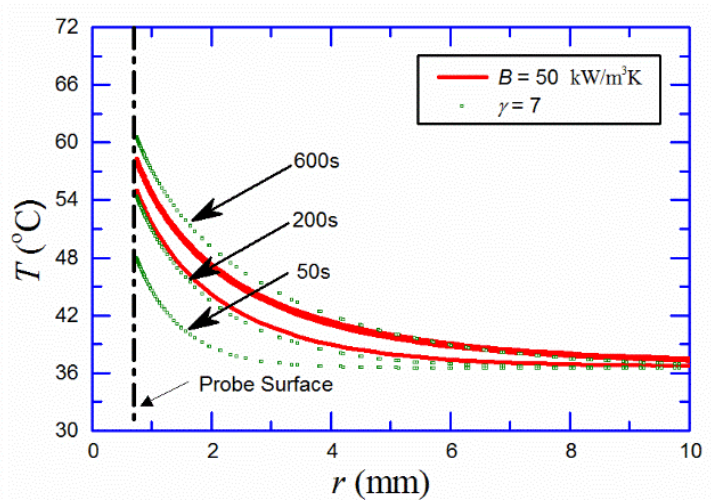

(b) $B=50 \mathrm{~kW} /\left(\mathrm{m}^{3} \cdot \mathrm{K}\right)$

Fig. 4 Change of temperature distribution at different rate of blood perfusion compared with the result of given apparent heat capacity in the case of heating at a constant heat flux of $10 \mathrm{~kW} / \mathrm{m}^{2}$.

\subsection{Freezing}

Figure 5 shows the effect of blood perfusion, thermal conductivity and heat capacity on the radial temperature distribution at the middle of cold probe at $600 \mathrm{~s}$ after the start of cooling to $\sim-90^{\circ} \mathrm{C}$. In all cases, the temperature gradient sharply changes at $-0.5^{\circ} \mathrm{C}$ at the edge of partially frozen region owing to the change of the thermal conductivity (see Appendix). However, the change is not clear at $-21.1^{\circ} \mathrm{C}$ where the tissue is completely frozen. The increase of blood perfusion makes the ice ball, i.e. the frozen region, smaller and the temperature gradient larger at the unfrozen region, which accordingly narrows the thermally affected region (Fig. 5(a)). The increase of heat capacity has the similar effect on the temperature distribution (Fig. 5(c)). In terms of the size of the ice ball, the effect of thermal conductivity is the same (Fig. 5(b)). However, because the temperature gradient is higher at lower thermal conductivities, the temperature distribution at the unfrozen region is significantly different. 


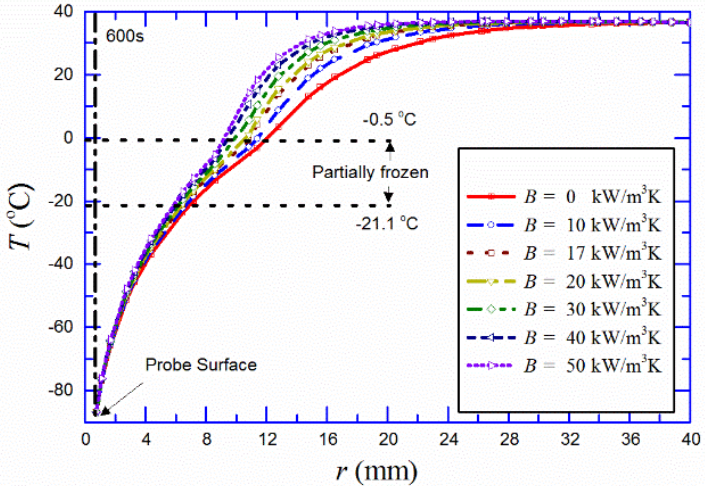

(a) Effect of the rate of blood perfusion

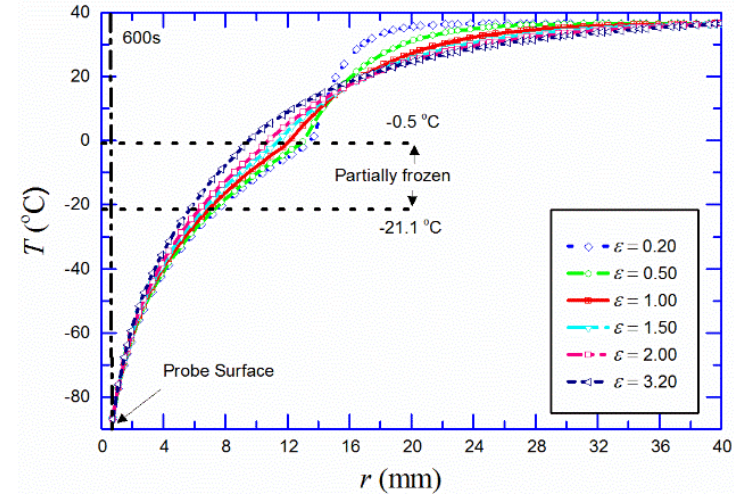

(b) Effect of apparent thermal conductivity

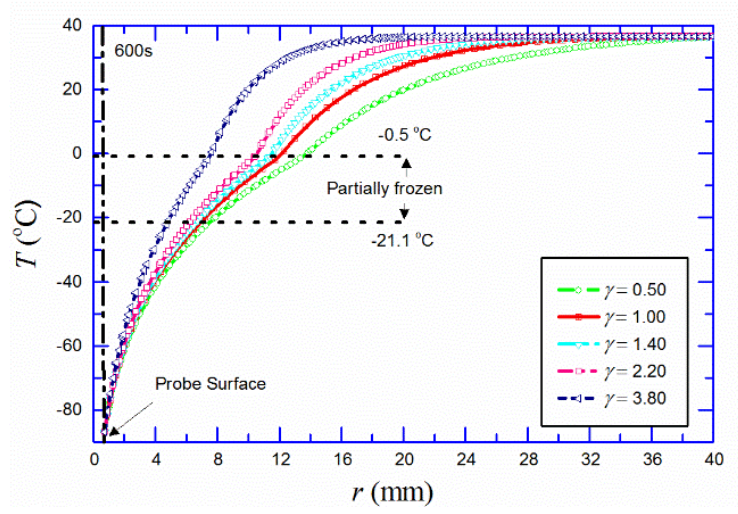

(c) Effect of apparent specific heat capacity

Fig. 5 Radial temperature distribution for the freezing case.

Figure 6 shows the temperature distribution at a given rate of blood perfusion compared with that for a specific apparent heat capacity. If we choose $\gamma=1.4$ for $B=17 \mathrm{~kW} /\left(\mathrm{m}^{3} \cdot \mathrm{K}\right)$ (Fig. 6 (a)) and $\gamma=2.2$ for $B=50$ $\mathrm{kW} /\left(\mathrm{m}^{3} \cdot \mathrm{K}\right)$ (Fig. 6(b)), the temperature distribution for given $B$ and $\gamma$ agrees well with one another at 200 and 400 $\mathrm{s}$ inside the ice ball. It was also the case for the different probe temperatures at different gas pressures (data not shown). However, at the unfrozen region, the temperature does not agree well with one another particularly at 600 s. A significant difference is also found near the surface of ice ball, i.e. the position at $-0.5^{\circ} \mathrm{C}$ at $600 \mathrm{~s}$.

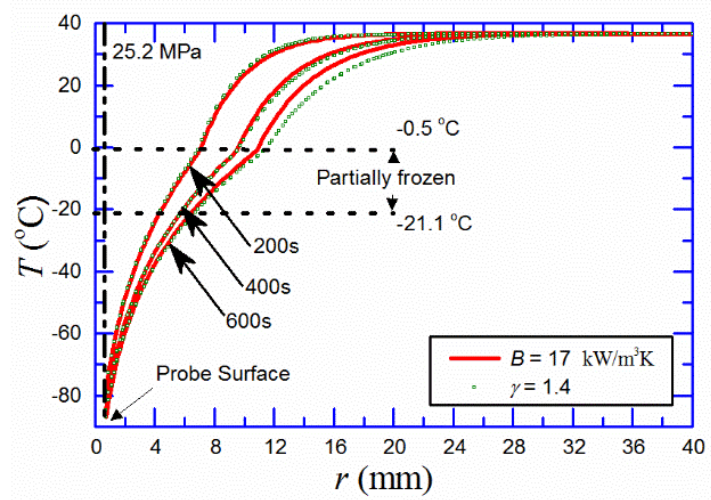

(a) $B=17 \mathrm{~kW} /\left(\mathrm{m}^{3} \cdot \mathrm{K}\right)$ and $\gamma=1.4$

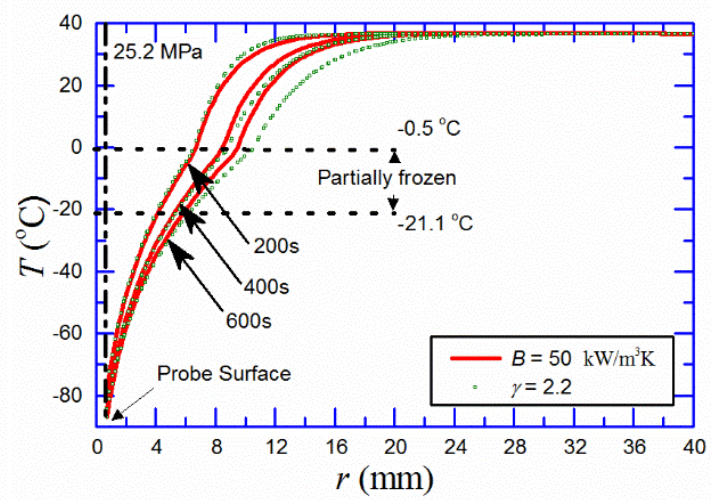

(b) $B=50 \mathrm{~kW} /\left(\mathrm{m}^{3} \cdot \mathrm{K}\right)$ and $\gamma=2.2$

Fig. 6 Comparison of the temperature distributions for a given rate of blood perfusion and that for a specific apparent heat capacity.

The data shown in Fig. 6 for given $B$ are compared with those obtained for a specific apparent thermal conductivity in Fig. 7. In this case, the value of $\varepsilon$ was selected focusing only on the temperature inside the ice ball. Although, the temperature distribution outside of the ice ball is not in agreement with one another, the 
temperature inside the ice ball for $\varepsilon=1.9$ and 2.9 agrees well with that for $B=17$ and $50 \mathrm{~kW} /\left(\mathrm{m}^{3} \cdot \mathrm{K}\right)$, respectively, irrespective of time.

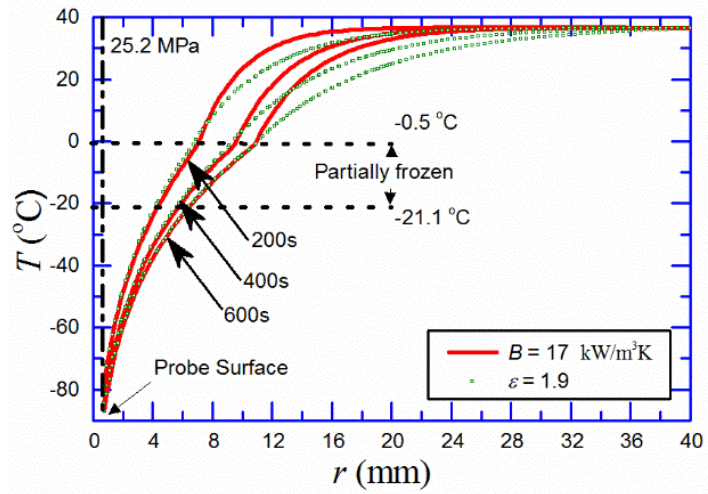

(a) $B=17 \mathrm{~kW} /\left(\mathrm{m}^{3} \cdot \mathrm{K}\right)$ and $\varepsilon=1.9$

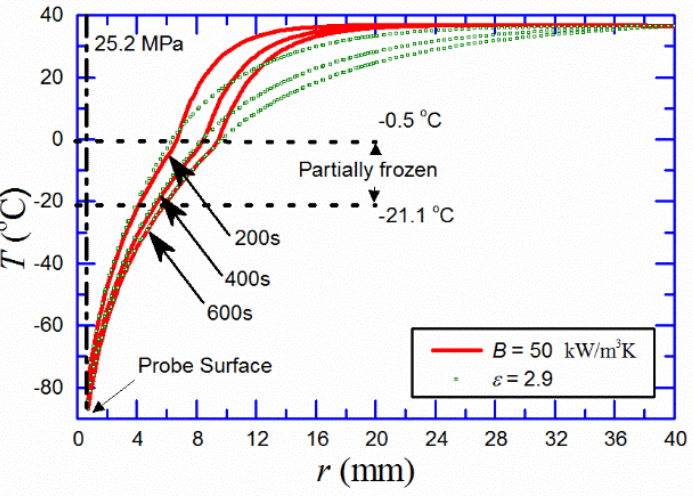

(b) $B=50 \mathrm{~kW} /\left(\mathrm{m}^{3} \cdot \mathrm{K}\right)$ and $\varepsilon=2.9$

Fig. 7 Comparison of the temperature distributions for a given rate of blood perfusion and that for a specific apparent thermal conductivity.

Similar comparison of the data for the other values of $B$ ranging from 10 to $50 \mathrm{~kW} /\left(\mathrm{m}^{3} \cdot \mathrm{K}\right)$ also demonstrated that the temperature distribution inside the ice ball for a given $B$ agrees well with that obtained with a specific value of $\varepsilon$. The value of $\varepsilon$ that gives the best-fit temperature distribution was constant irrespective of time as shown in Fig. 7. We also determined the optimal value of $\varepsilon$ for different probe temperatures resulting from different gas pressures. Thus determined values are summarized in Fig. 8. The optimum value of $\varepsilon$ is larger for larger $B$ but is independent of the probe temperature resulted from different gas pressure.

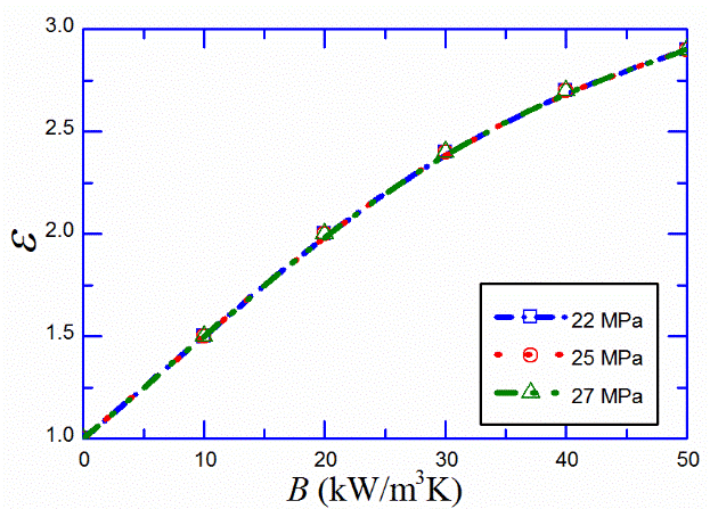

Fig. 8 The best-fit multiplier for apparent thermal conductivity as a function of blood perfusion rate.

Figure 9 shows the comparison of the results for a given $B$ and the best-fit $\varepsilon$ in terms of the growth of ice ball. The radius of ice ball for $B=17 \mathrm{~kW} /\left(\mathrm{m}^{3} \cdot \mathrm{K}\right)$ agrees with that for $\varepsilon=1.9$ within $5 \%$ difference at any gas pressure during the whole process of freezing. The results of $B=50 \mathrm{~kW} /\left(\mathrm{m}^{3} \cdot \mathrm{K}\right)$ and $\varepsilon=2.9$ also agrees with one another within $6 \%$ difference irrespective of time and the gas pressure. 


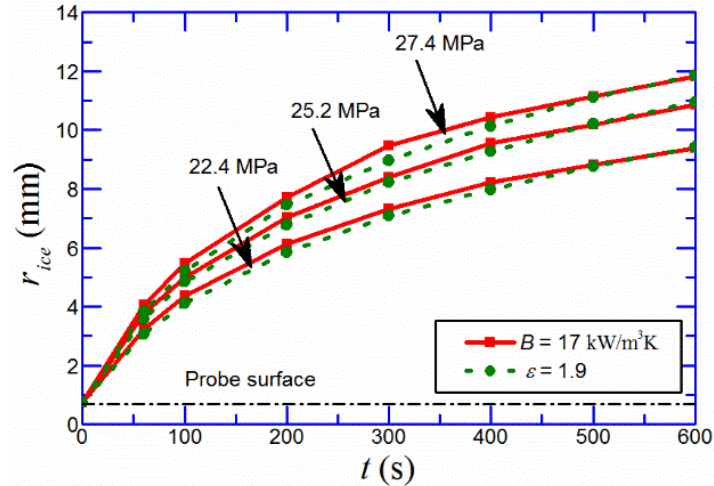

(a) $B=17 \mathrm{~kW} /\left(\mathrm{m}^{3} \cdot \mathrm{K}\right)$ and $\varepsilon=1.9$

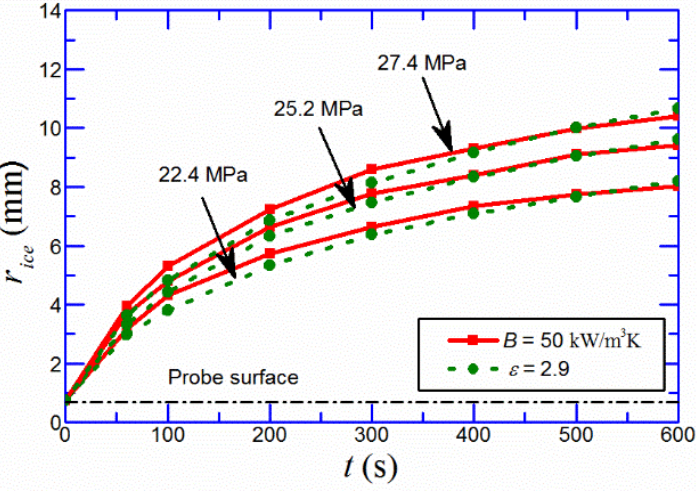

(b) $B=50 \mathrm{~kW} /\left(\mathrm{m}^{3} \cdot \mathrm{K}\right)$ and $\varepsilon=2.9$

Fig. 9 The growth of ice ball at a given rate of blood perfusion and a corresponding apparent thermal conductivity

\section{Discussion}

Taking the effect of blood perfusion into account is essential when dealing with heat transport in tissues or organs in vivo. The rate of blood perfusion $\omega_{b}(1 / \mathrm{s})$ in the bioheat equation is the volumetric rate of blood flow per unit volume of tissue, which is not a physical quantity measured using commercially available blood flowmeters. Therefore, the value of $\omega_{b}$ is not available in many cases; particularly in human tissues. The motivation of the present study is therefore to use the normal heat conduction equation with apparent thermophysical properties instead of using the bioheat equation. Although this idea seems to be a practical and simple alternative to incorporate the effect of blood flow, as far as the authors know, no papers clearly showed the difference in the temperature distribution between those calculated from the heat conduction equation and the bioheat equation.

The rate of heat transfer is obviously increased by blood perfusion. The higher thermal conductivity also makes the heat transfer rate higher for a given temperature difference. In the case of transient heat conduction, the heat transfer rate is higher at higher heat capacities. These are the reason why the thermal conductivity and the heat capacity were examined as the potential apparent thermophysical properties. However, in terms of the temperature distribution for a given probe temperature, increasing thermal conductivity reduces the temperature gradient, while increasing the blood perfusion has the opposite effect (Fig. 3(a)). In contrast, the effect of increasing the heat capacity is similar to that of blood perfusion in the case where the probe temperature is changed to a given temperature (Fig. 3(b)). However, it is not the case for the given heat flux condition (Fig. 4). Consequently, the blood perfusion term in the bioheat equation cannot be replaced by the apparent thermal conductivity or the apparent heat capacity to estimate the temperature distribution.

However, the present study demonstrated a case where the apparent thermal conductivity is useful for taking the effect of blood perfusion into account. One of the most important issues in pre-operation planning of cryosurgery is predicting the size of the ice ball. This is why the freezing during cryosurgery was taken up for examination in the present study. If we are interested only in the size of the ice ball, we are able to incorporate the effect of blood perfusion by introducing the apparent thermal conductivity. A unique value of a multiplier to the original thermal conductivity was determined to predict the size of the growing ice ball for a given rate of blood perfusion irrespective of time and probe temperature. The successful result in the freezing case owes to the fact that no blood perfusion exists in the ice, which results in no difference in the thermophysical properties in the frozen part irrespective of blood perfusion in the tissue. Therefore, the increase of apparent thermal conductivity only influences the heat transfer from the surface of ice ball to the outside.

Another issue that is critically important in operating cryosurgery is estimation of a proper excess margin of the ice ball to completely destroy undesirable cancerous tissues. Cancer cells are necrotized when they are frozen at lower than their lethal temperature that is normally considered to be $-20^{\circ} \mathrm{C}$ or $-40^{\circ} \mathrm{C}$ depending on cell types (Chosy et al., 1997). We therefore need to make the ice ball larger than the target with some excess margins at the ice periphery. However, too large a margin induces destruction of a large amount of healthy normal cells. On the 
contrary, insufficient margin could induce the recurrence of cancer. It is therefore important to estimate the proper margin for given conditions. By conducting experiments and a simulation for a tissue phantom with no blood perfusion, the authors have proposed a guideline for the safety margin that depends on the size of ice ball (Shurrab et al., 2016). However, to make the guideline more reliable, we need to confirm that the temperature distribution inside the ice ball is almost independent of the blood perfusion. Figure 10 shows the temperature distribution inside the ice ball of the same size ( $~ 8 \mathrm{~mm}$ in radius) at different rate of blood perfusion. It should be noted that the results for different blood perfusion are those at different time because longer time is needed for producing the ice ball of the same size at larger rate of blood perfusion. It is obvious that the temperature distribution inside the ice ball agrees between different blood perfusion rates. Therefore, the guideline we have previously proposed about the safety margin is useful irrespective of the rate of blood perfusion. Consequently, the apparent thermal conductivity is also useful for determination of the safety margin.

In the present study, we determined the multiplier for the thermal conductivity in the heat conduction equation by comparing the temperature distribution inside the ice ball with that calculated from the bioheat equation. This was based on the hypothesis that the bioheat equation provides actual temperature distribution inside living tissues. However, there is only a few studies, including the first paper by Pennes (Pennes, 1948), which measured temperature distribution in vivo. Therefore, we expect to have non-invasive methods for measuring temperature distribution in living tissues, which definitely contributes to medical treatments.

Measurement of thermophysical properties of living tissues is essential even if their apparent values work for including the effect of blood perfusion. However, this is actually difficult because non-invasive technique is required for in-vivo measurement particularly for human tissues. This is one of the motivations of our study that proposed apparent thermophysical properties for practical use. We can use the multiplier defined in this study together with thermophysical properties that have been measured in vitro by conventional methods with 'dead' tissues. However, estimation of the multiplier is still needed based on experiments. One of the ways proposed here is to determine from real cryosurgeries. In the state-of-the-art cryosurgery, the ice ball is monitored in-situ using MRI. Therefore, in the similar way as this study, the apparent thermal conductivity can be determined from the size of ice ball collected from these clinical data. We can thus accumulate data of apparent thermal conductivity for a variety of tissues and organs. The database will be useful for prediction of ice ball or the safety margin in many facilities even with little experience of cryosurgery. The advantage of using apparent thermal conductivity is that we can use most commercially available software to solve the heat conduction equation instead of the bioheat equation that is not included in many cases.

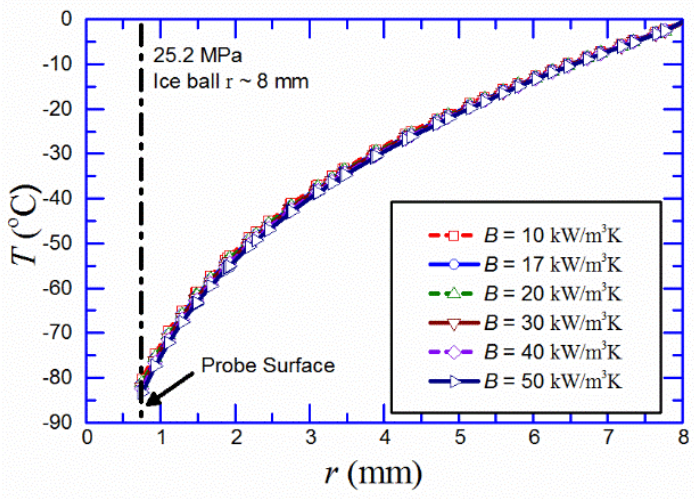

Fig. 10 The radial temperature distribution inside an ice ball of the same radius at different blood perfusion

\section{Conclusions}

The effect of blood perfusion that is taken into account in the bioheat equation cannot be expressed by the apparent thermal conductivity or the apparent heat capacity in general for the purpose of estimating the temperature distribution in tissues. However, the apparent thermal conductivity is useful for estimation of the size of ice ball produced in cryosurgery. The apparent thermal conductivity is uniquely determined for a given rate of blood perfusion irrespective of probe temperature and time, and increases with the blood perfusion rate. For practical use, the value of apparent thermal conductivity is to be determined from measured size of the ice ball 
during cryosurgery.

\section{Appendix}

The thermal conductivity used in this study is shown in Fig. A1 as a function of temperature (see Ref. (Shurrab et al., 2016) for the estimation method). The effect of blood perfusion is included in the apparent thermal conductivity only at the unfrozen part because no blood flow was assumed at the partially and fully frozen part.

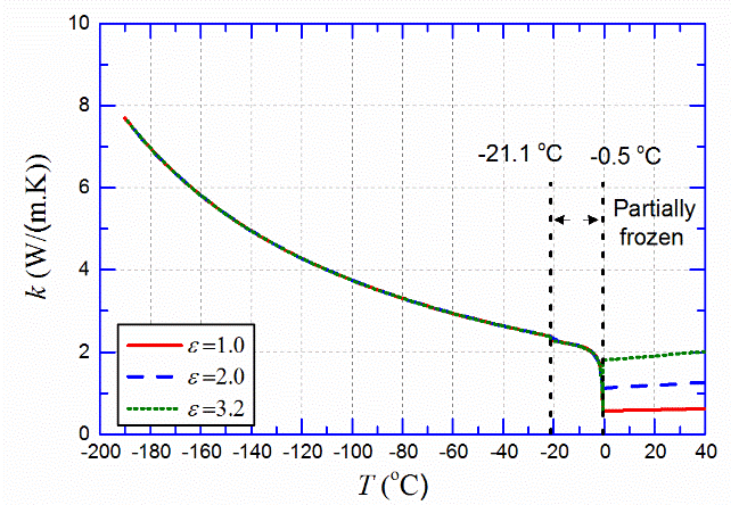

Fig. A1 Thermal conductivity as a function of temperature.

\section{References}

Baish, J. W., Heat transport by countercurrent blood vessels in the presence of an arbitrary temperature gradient, Journal of Biomechanical Engineering, Vol. 112, No. 2 (1990), pp.207-211.

Bowman, M. F., The simultaneous measurement of thermal conductivity, thermal diffusivity, and perfusion in small volumes of tissue, Journal of Biomechanical Engineering, Vol. 106, No. 3 (1984), pp.192-197.

Chato, J. C., Heat transfer to blood vessels, ASME Journal of Biomechanical Engineering, Vol. 102, No. 2 (1980), pp.110-118.

Chen, M. M., and Holmes, K. R., Microvascular contributions in tissue heat transfer, Annals of the New York Academy of Sciences, Vol. 335, No. 1 (1980), pp.137-150.

Chosy, S. G., Nakada, S. Y., Lee, F. T., and Warner, T. F., Monitoring renal cryosurgery: predictors of tissue necrosis in swine, The Journal of urology, Vol. 159, No. 4 (1998), pp.1370-1374.

Dehghan, M., and Sabouri, M., A spectral element method for solving the Pennes bio-heat transfer equation by using triangular and quadrilateral elements, Applied Mathematical Modelling, Vol. 36, No. 12 (2012), pp.6031-6049.

Diller, K. R., Valvano, J. W., and Pearce, J. A., Bioheat transfer (1999) In CRC handbook of thermal engineering, pp. 4-114, CRC Press.

Duck F., Physical properties of tissues: a comprehensive reference book (1990). Academic press.

Gang Z., Zhifeng L., and Dayong G., Effect of temperature dependent blood perfusion and metabolic heat generation on heat transfer problem in cryosurgery, Journal of Chemical Industry and Engineering, Vol. 58, No. 5 (2007), pp.1144-1144.

Huang, H. W., Chen, Z. P., and Roemer, R. B., A counter current vascular network model of heat transfer in tissues, ASME Journal of Biomechanical Engineering, Vol. 118, No. 1 (1996), pp.120-129.

Jain, M. K., and Wolf, P. D., A three-dimensional finite element model of radiofrequency ablation with blood flow and its experimental validation, Annals of Biomedical Engineering, Vol. 28, No. 9 (2000), pp.1075-1084.

Jiji, L. M., Weinbaum, S., and Lemons, D. E., Theory and experiment for the effect of vascular microstructure on surface tissue heat transfer-part II: model formulation and solution, Journal of Biomechanical Engineering, Vol. 106, No. 4 (1984), pp.331-341. 
Koehler, R. C., Traystman, R. J., and Jones, M. D., Regional blood flow and O2 transport during hypoxic and CO hypoxia in neonatal and adult sheep, American Journal of Physiology-Heart and Circulatory Physiology, Vol. 248, No. 1 (1985), pp.H118-H124.

Lemons, D.E., Weinbaum, S., and Jiji, L.M., Experimental studies on the role of the micro and macro vascular system in tissue heat transfer, American Journal of Physiology, Vol. 253, (1987), R128.

Li H., Xuexue Z., and Jing L., Heat Transfer Analysis on Laser-tissue Thermal Interaction Using Heterogeneous Model, Chinese Journal of Laser, Vol. 11, No. 3 (2002), pp.233-238.

Maass-Moreno, R., Damianou, C. A., and Sanghvi, N. T., Noninvasive temperature estimation in tissue via ultrasound echo-shifts. Part II. Invitro study, The Journal of the Acoustical Society of America, Vol. 100, No. 4 (1996), pp.2522-2530.

Mooibroek, J., and Lagendijk, J. J. W., A fast and simple algorithm for the calculation of convective heat transfer by large vessels in three-dimensional inhomogeneous tissues, IEEE Transactions on Biomedical Engineering, Vol. 38, No. 5 (1991), pp.490-501.

Newman, W. H., and Lele, P. P., A transient heating technique for the measurement of thermal properties of perfused biological tissue, Journal of Biomechanical Engineering, Vol. 107, No. 3 (1985), pp.219-227.

Oleson, J. R., Dewhirst, M. W., Harrelson, J. M., Leopold, K. A., Samulski, T. V., and Tso, C. Y., Tumor temperature distributions predict hyperthermia effect, International Journal of Radiation Oncology* Biology* Physics, Vol. 16, No. 3 (1989), pp.559-570.

Pennes, H. H., Analysis of tissue and arterial blood temperatures in the resting human forearm, Journal of Applied Physiology, Vol. 1, No. 2 (1948), pp.93-122.

Sanyal, D., and Maji, N., Thermoregulation through skin under variable atmospheric and physiological conditions, Journal of Theoretical Biology, Vol. 208, No. 4 (2001), pp.451-456.

Seyde, W. C., McGowan, L., Lund, N., Duling, B., and Longnecker, D. E., Effects of anesthetics on regional hemodynamics in normovolemic and hemorrhaged rats, American Journal of Physiology-Heart and Circulatory Physiology, Vol. 249, No. 1 (1985), pp.H164-H173.

Shahnazari, M., Aghanajafi, C., Azimifar, M., and Jamali, H., Investigation of bio-heat transfer equation of pennes via a new method based on wrm and homotopy perturbation, International Journal of Research and Reviews in Applied Sciences, Vol. 17, No. 3 (2013).

Shitzer, A., and Eberhart, R., Heat Transfer in Medicine and Biology. Plenum Publishing Corporation (1985).

Shurrab, M., Wang, H., Kubo, N., Fukunaga, T., Kurata, K., and Takamatsu, H., The cooling performance of a cryoprobe: Establishing guidelines for the safety margins in cryosurgery, International Journal of Refrigeration, Vol. 67, (2016), pp.308-318.

Valvano, J. W., Bioheat transfer, Encyclopedia of Medical Devices and Instrumentation (2005).

Valvano, J. W., Allen, J. T., Walsh, J. T., Hnatowich, D. J., Tomera, J. F., Brunengraber, H., and Bowman, H. F., An isolated rat liver model for the evaluation of thermal techniques to quantify perfusion, Journal of Biomechanical Engineering, Vol. 106, No. 3 (1984), pp.187-191.

Weinbaum, S. and Jiji, L.M., A new simplified bioheat equation for the effect of blood flow on average tissue temperature, Journal of Biomechanical Engineering, Vol. 107, No. 2 (1985), pp.131-139.

Weinbaum, S. J. L. M., Jiji, L. M., and Lemons, D. E., Theory and experiment for the effect of vascular microstructure on surface tissue heat transfer-Part I: Anatomical foundation and model conceptualization, Journal of Biomechanical Engineering, Vol. 106, No. 4 (1984), pp.321-330.

Xu, L. X., The Theoretical Evaluation of the Pennes, the Chen-Holmes and the Weinbaum-Jiji Bioheat Transfer Models in the Pig Renal Cortex, ASME Winter Annual Meeting, Atlanta, HTD Vol. 189, (1991), pp. 15-22.

Zhao G., Zhang H., Guo X., Luo D., and Gao D., Effect of blood flow and metabolism on multidimensional heat transfer during cryosurgery, Medical Engineering and Physics, Vol. 29, No. 2 (2007), pp.205-215.

Zhao, J. J., Zhang, J., Kang, N., and Yang, F., A two level finite difference scheme for one dimensional Pennes' bio-heat equation, Applied Mathematics and Computation, Vol. 171, No. 1 (2005), pp.320-331. 